\title{
Refractory Primary Hepatic Actinomycosis with Direct Infiltration to the Diaphragm and Thorax: The Usefulness of Contrast-enhanced Ultrasonography
}

\author{
Shinji Oe ${ }^{1}$, Michihiko Shibata ${ }^{1}$, Masaaki Hiura ${ }^{1}$, Hiroshi Mitsuoka ${ }^{2}$, Toru Matsuhashi ${ }^{1}$, \\ Ryoichi Narita ${ }^{1,3}$, Shintaro Abe ${ }^{1}$, Akinari Tabaru ${ }^{1}$, Kazuhiro Hayashida ${ }^{2}$, \\ Hatsumi Taniguchi ${ }^{4}$ and Masaru Harada ${ }^{1}$
}

\begin{abstract}
An 80-year-old man was admitted to our hospital with a diagnosis of primary hepatic actinomycosis determined based on a percutaneous aspiration biopsy. The abscesses and state of liquefaction were easily and effectively visualized on contrast-enhanced ultrasonography. Ampicillin/sulbactam was administered; however, lesions of hepatic actinomycosis suddenly infiltrated the diaphragm and right thorax six months later. A drainage tube was inserted into the right thoracic space, and the pleural effusion gradually decreased. The patient received continuous antibiotic therapy for nearly two years and remained free of hepatic actinomycosis on follow-up more than one year later.
\end{abstract}

Key words: hepatic actinomycosis, hepatic abscess, contrast-enhanced ultrasonography (CEUS), diaphragm, thorax

(Intern Med 53: 2073-2078, 2014)

(DOI: 10.2169/internalmedicine.53.2261)

\section{Introduction}

Actinomycosis is a rare and chronic pyogenic granulomatous disease caused by infection with the filamentous Grampositive anaerobic bacteria, Actinomyces israelii, which is part of the normal flora of the oropharynx, gastrointestinal tract and urogenital tract, with the disease occurring in these areas (1-3). Hepatic actinomycosis is caused by direct invasion or hematogenous spread via the portal vein from sites such as the appendix and ileocecum. The condition is called primary hepatic actinomycosis if there are no other primary lesions (4). Accurately diagnosing primary hepatic actinomycosis is difficult, and many cases are misdiagnosed as ordinary bacterial abscesses or malignant tumors and treated with non-sensitive antibiotics or hepatectomy, respectively. We herein report a case of primary hepatic actinomycosis that was diagnosed on a needle aspiration biopsy and which directly infiltrated the diaphragm and right thorax five months after the induction of treatment, despite the use of sensitive antibiotics.

\section{Case Report}

An 80-year-old man who had been taking prednisolone at a dose of at least $10 \mathrm{mg} /$ day for polymyalgia rheumatica for two years was admitted to our hospital for the first time in 2010 with a four-month history of a high fever and right upper abdominal pain. He had undergone laparoscopic partial gastrectomy for early gastric cancer and percutaneous transhepatic gallbladder drainage for acute cholecystitis in 2005 . A physical examination showed tenderness in the upper right abdomen; however, no arthralgia or myalgia were detected. The laboratory data showed inflammation and renal

\footnotetext{
${ }^{1}$ The Third Department of Internal Medicine, School of Medicine, University of Occupational and Environmental Health, Japan, ${ }^{2}$ Department of Internal Medicine, JR Kyushu Hospital, Japan, ${ }^{3}$ Department of Gastroenterology, Oita Red Cross Hospital, Japan and ${ }^{4}$ Department of Microbiology, School of Medicine, University of Occupational and Environmental Health, Japan
} Received for publication December 9, 2013; Accepted for publication March 11, 2014 Correspondence to Dr. Shinji Oe, ooes@med.uoeh-u.ac.jp 

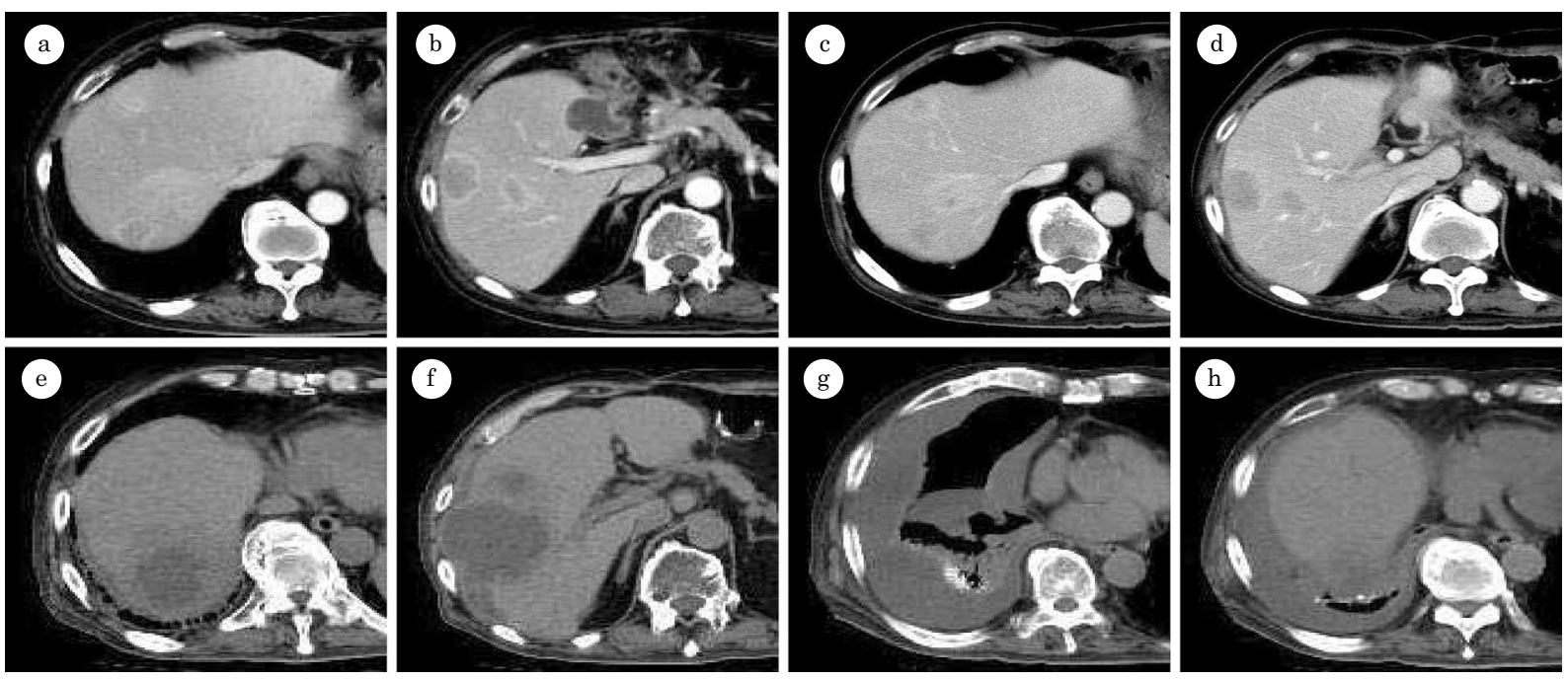

Figure 1. Contrast-enhanced computed tomography (CT) performed on the first admission showed multiple solid ring-enhancing masses in the right hepatic lobe (a, b). Although the liver abscesses were found to have decreased in size and number two months after the induction of treatment (c, d), they had increased again at five months after the start of treatment (e, f). At six months after the initiation of treatment, encapsulated pleural effusion appeared suddenly in the right thorax, and the liver abscess under the diaphragm collapsed and decreased in size $(\mathrm{g}, \mathrm{h})$.

dysfunction, and he was diagnosed with diabetes based on an HbA1c level of 7.2\% (National Glycohemoglobin Standardization Program). Contrast-enhanced computed tomography (CT) showed multiple ring-enhancing solid masses in the right hepatic lobe with a maximum size of $4 \mathrm{~cm}$ in diameter (Fig. 1a, b). These lesions were vague and hypoechoic on B-mode ultrasonography, although they were clearly visualized in the Kupffer phase of perflubutane contrast-enhanced ultrasonography (CEUS) (Fig. 2a, c). We confirmed that the liver masses were not liquefied, because all masses were generally enhanced as hypervascular during the arterial phase of CEUS (Fig. 2b). The patient was diagnosed with bacterial liver abscesses and administered empirical intravenous imipenem/cilastatin (IPM/CS). However, his clinical course differed from that of usual bacterial liver abscesses, as his fever did not improve and the lesions had not liquefied one month after the induction of treatment. Therefore, we performed an ultrasonography-guided percutaneous aspiration biopsy of a hepatic nodule using a 21-G needle. The pathology specimen showed an abscess-forming dense acute inflammatory infiltrate and colonies of Actinomyces with tiny radiate granules and peripheral eosinophilic clubs (Fig. 3). Hence, we made a diagnosis of primary hepatic actinomycosis and changed the antibiotic from IPM/CS to ampicillin/sulbactam (ABPC/SBT) at a dose of $1.5 \mathrm{~g}$ administered twice a day. The patient's fever and abdominal pain rapidly improved, and his white blood cell count and $\mathrm{C}$ reactive protein concentration decreased (Fig. 4). Two months after the initiation of treatment, the liver abscesses had decreased in both size and number on CT (Fig. 1c, d). Therefore, the intravenous ABPC/SBT therapy was changed to oral amoxicillin (AMPC) at a dose of $750 \mathrm{mg} / \mathrm{day}$, and the patient was discharged. Five months after the start of treatment, the hepatic actinomycotic abscesses had recovered in both size and number on CT (Fig. 1e, f) following the switch of AMPC to erythromycin (EM) at a dose of 750 $\mathrm{mg} /$ day due to suspicion of an allergic skin eruption. Moreover, the central area of the liver masses exhibited no blood flow during the arterial phase of CEUS, which indicated liquefaction of the abscesses (Fig. 2d, e). However, one of the liver abscesses located just under the diaphragm had increased especially rapidly (Fig. 1e), while another lesion that had been punctured for a biopsy was found to be growing outside the liver along the puncture line (Fig. 1f). The laboratory data also showed an increase in inflammation, with a white blood cell count of $20,000 / \mu \mathrm{L}$ with neutrophilia and a C-reactive protein concentration of $18.0 \mathrm{mg} / \mathrm{dL}$ (Fig. 4). Therefore, the patient was readmitted to our hospital and treated again with ABPC/SBT at a dose of $1.5 \mathrm{~g}$ twice a day. Although the inflammation gradually improved, he suddenly complained of dyspnea at six months after the induction of treatment. Encapsulated pleural effusion appeared in the right thorax, and the liver abscess under the diaphragm collapsed (Fig. 1g, h, Fig. 5). The patient was diagnosed with hepatic actinomycosis infiltrating directly to the diaphragm and right thorax. A chest tube was subsequently inserted into the right thoracic space, and the serous pleural effusion was drained continuously. The dose of ABPC/SBT was also increased to $1.5 \mathrm{~g}$ three times a day. The pleural effusion decreased gradually, and the chest tube was removed seven months after the start of treatment. The patient continued to receive intravenous AMPC/SBT, and the liver abscesses and extrahepatic lesions improved very slowly. He ultimately received continuous antibiotic therapy for approximately two years, as small low-density areas in the liver persisted on CT. Although he has since taken ster- 

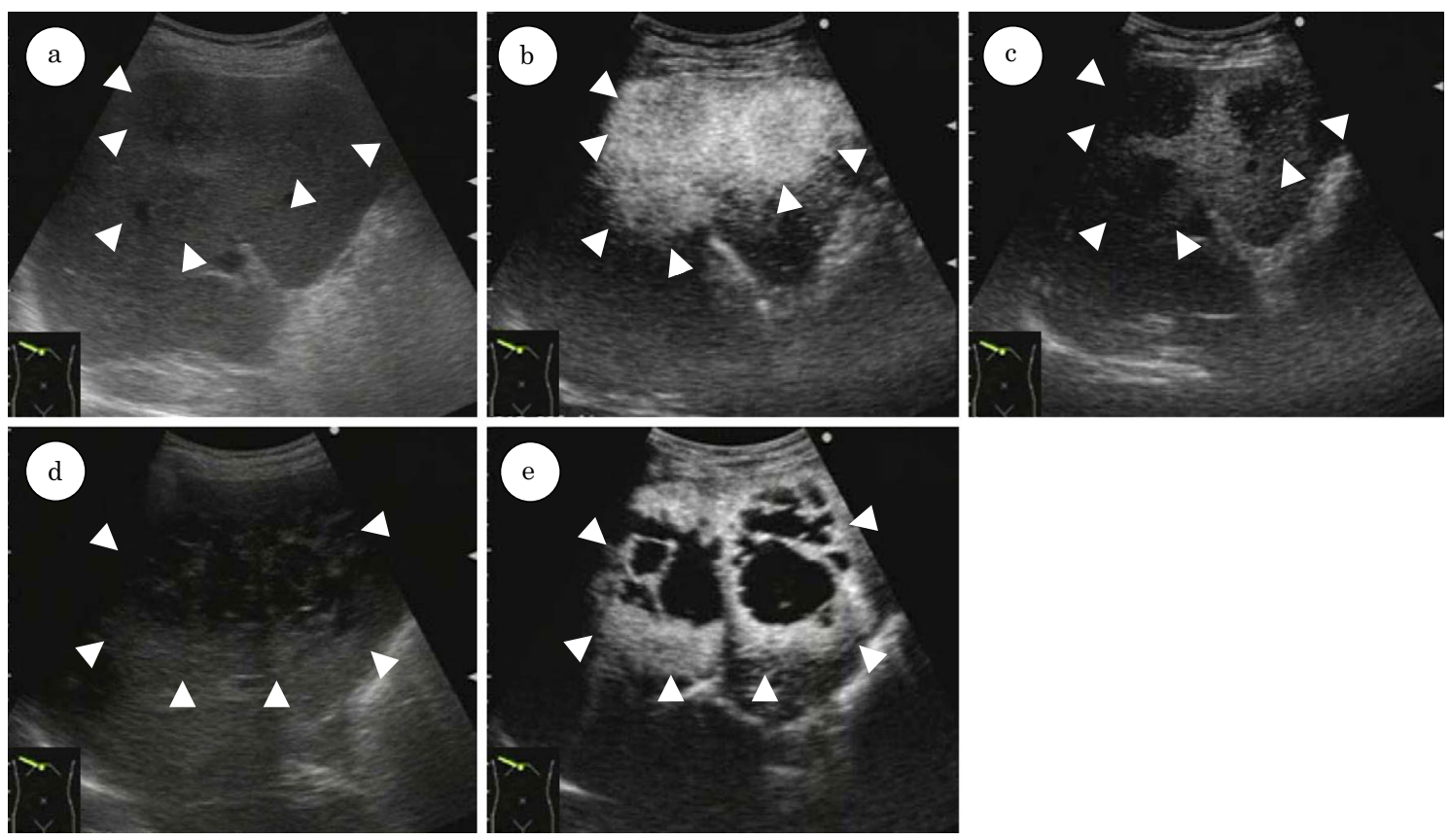

Figure 2. B-mode ultrasonography performed on the first admission showed vague and hypoechoic masses (arrowheads) (a). In contrast, perflubutane contrast-enhanced ultrasonography (CEUS) revealed enhanced masses (arrowheads) as hypervascular lesions during the arterial phase within 13 seconds after the venous injection of $\operatorname{Sonazoid}^{\circledR}(\mathrm{b})$ and clearly visualized hypoechoic masses (arrowheads) during the Kupffer phase within 14 minutes after administration (c). Moreover, five months after the start of treatment, the central areas of liver masses (arrowheads) had no blood flow during the arterial phase of CEUS within 19 seconds after administration, which indicated liquefaction of the abscesses (d, e).

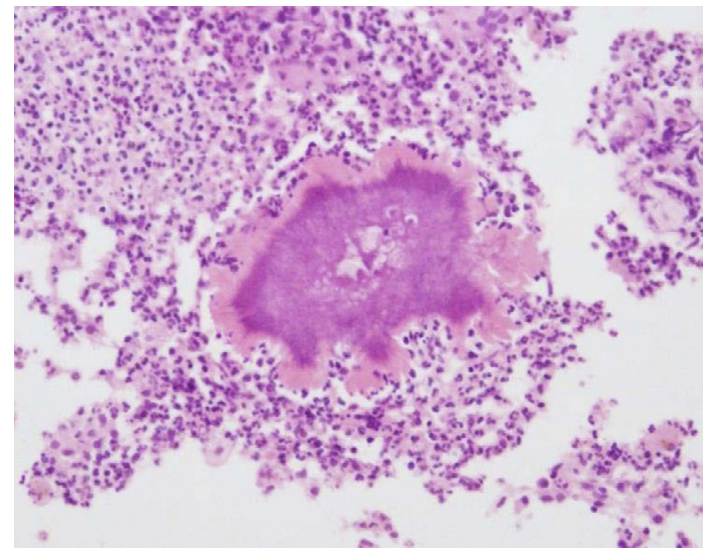

Figure 3. Histological examination of the hepatic nodule specimen obtained via an aspiration needle biopsy. The pathology specimen showed abscess-forming dense acute inflammatory infiltrates and Actinomyces colonies with tiny radiate granules and peripheral eosinophilic clubs.

oids for polymyalgia rheumatica, the hepatic actinomycosis has not recurred for more than one year.

\section{Discussion}

Actinomycosis occurs in the cervicofacial area (50\%), thoracic area $(15 \%)$ and abdominal area $(20 \%)(5)$. Hepatic actinomycosis accounts for approximately $15 \%$ of cases of abdominal actinomycosis and 5\% of all cases of actinomycosis. Primary hepatic actinomycosis is very rare (6). Sharma et al. reviewed 56 cases of hepatic actinomycosis and reported that the patients exhibited a wide age range (486 years) and were predominantly men (70.2\%) (7).

It is well known that a previous history of abdominal surgery, abdominal trauma and/or the presence of gastrointestinal foreign bodies are risk factors for abdominal actinomycosis (1). An immunosuppressed status, including that due to diabetes, chemotherapy or immunosuppressive therapy, is a predisposing condition for actinomycosis $(8,9)$. Although hepatic actinomycosis is thought to occur via the portal vein after mucosal injury from ulcers, inflammatory disease or surgery, it is difficult to isolate the primary focus (10). The present patient had a history of gastric surgery, percutaneous transhepatic gallbladder drainage and steroid treatment for polymyalgia rheumatica. In addition, he was diagnosed with diabetes mellitus on the first admission. Therefore, he was at high risk for hepatic actinomycosis.

The symptoms of hepatic actinomycosis include fever, weight loss and abdominal pain, although they are all nonspecific for the disease (11). It is also very difficult to distinguish the lesions from ordinary bacterial abscesses or malignant tumors on CT or magnetic resonance imaging, because the findings of both modalities are usually nonspecific and non-diagnostic $(6,7,12-14)$. We attempted to evaluate the lesions of hepatic actinomycosis using CEUS 


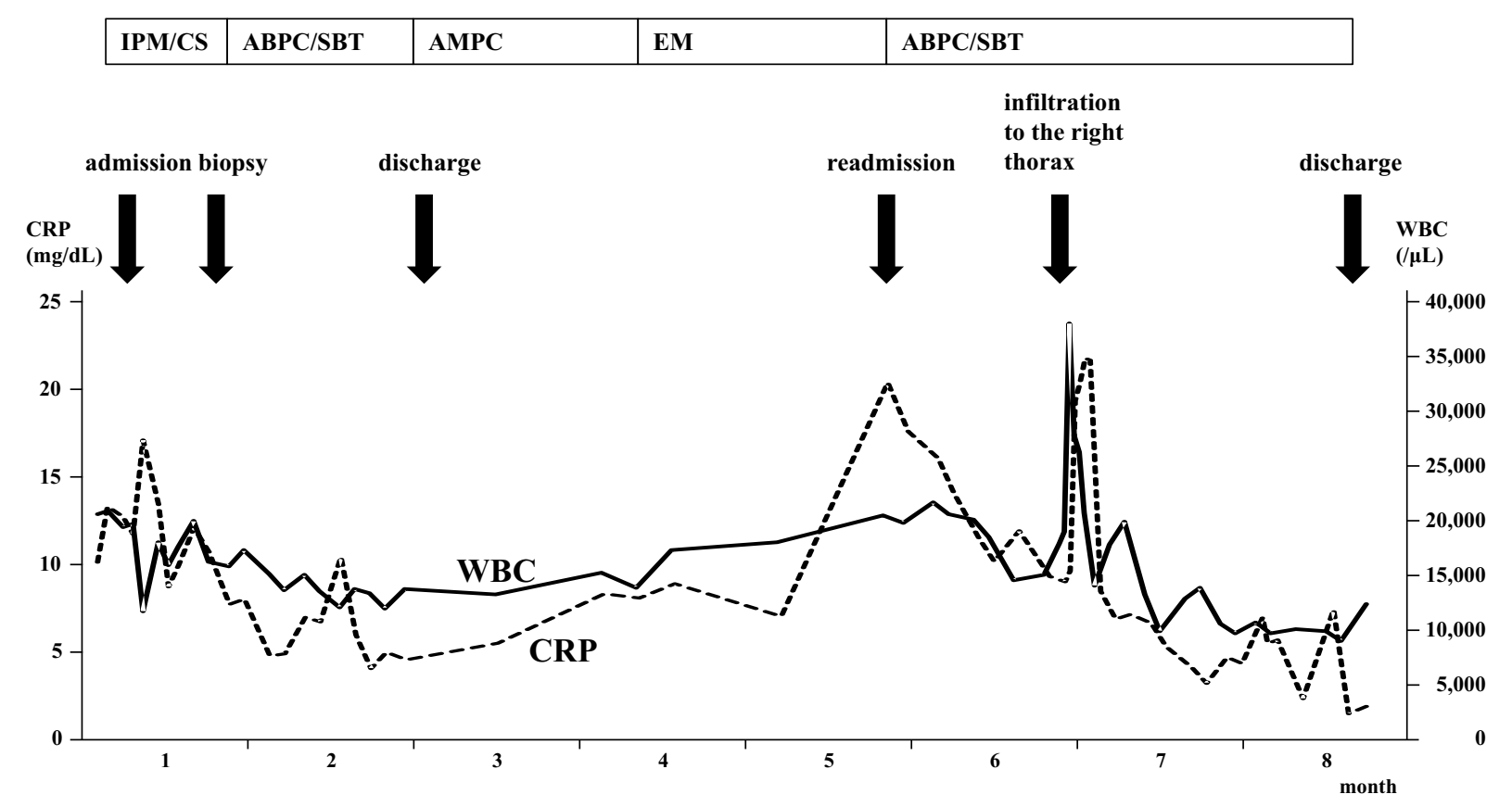

Figure 4. The patient's clinical course demonstrating an inflammatory response. The y-axes show the $\mathrm{C}$ reactive protein $(\mathrm{CRP})$ level $[\mathrm{mg} / \mathrm{dL}]$ and white blood cell $(\mathrm{WBC})$ count $[/ \mu \mathrm{L}]$. IPM/CS: imipenem/cilastatin, ABPC/SBT: ampicillin/sulbactam, AMPC: amoxicillin, EM: erythromycin

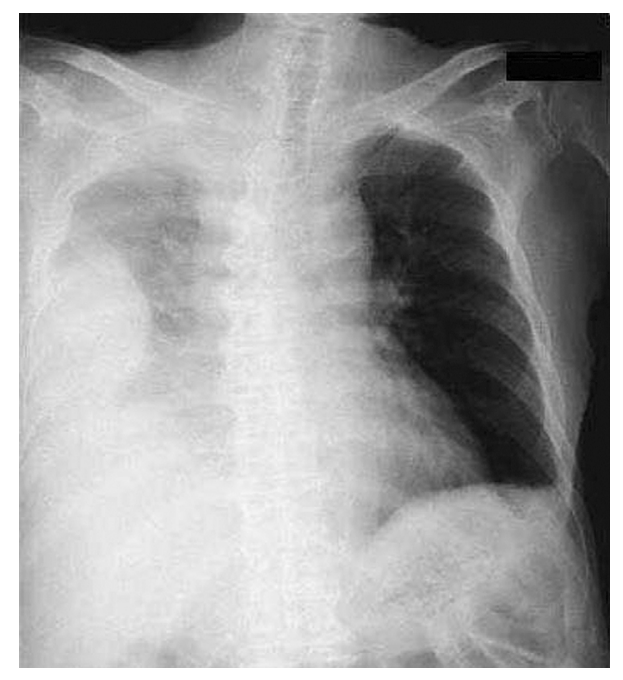

Figure 5. A chest radiograph showed encapsulated pleural effusion in the right thorax.

several times. Recently, CEUS has been used in the diagnosis of liver tumors, particularly hepatocellular carcinoma, and has been applied in the evaluation of liver abscesses $(15,16)$. The vascular images on CEUS of pyogenic liver abscesses, including that observed in our case, are typically hypervascular and very similar to CT findings. However, all abscesses, including ring-enhancing solid masses on CT, change gradually from hypervascular in the arterial phase to ring-enhancing in the portal phase on CEUS. Because CEUS is superior in temporal resolution, it is possible to observe the vascularity of the target continually in real time. On the other hand, CT has limitations in temporal resolution, and the quality of the vascular image of the mass depends on the timing of the scan. Therefore, the ringenhancing images of the abscesses on CT observed in our case may have been scanned in the portal phase, not the arterial phase. Although the areas of hepatic actinomycosis were vague on B-mode ultrasonography in the present case, they were clearly visualized during the Kupffer phase of CEUS. Moreover, it is often difficult to judge the liquefaction of liver abscesses for drainage. However, we were able to easily evaluate the liquefaction of the hepatic actinomycosis in both the presence and absence of a micro blood flow on CEUS (Fig. 2b, e).

The hepatic actinomycosis observed in the present case had not liquefied after one month of treatment. Therefore, it is an important feature that hepatic actinomycosis is often difficult to liquefy. This is the first report to describe the usefulness of CEUS for diagnosing hepatic actinomycosis. In this case, CEUS was very useful for visualizing the lesions and evaluating central liquefaction during the arterial phase.

We confirmed the liquefaction of the hepatic actinomycosis on CEUS five months after the start of treatment. However, we did not insert a drainage tube because Actinomyces tends to infiltrate locally to neighboring organs (17). In fact, one liver abscess in our case had grown outside the liver along the puncture line. Puncturing hepatic actinomycosis nodules should be avoided, except for the purpose of diagnosis.

Obtaining a definitive diagnosis depends on identifying actinomyces using histopathology or culture (1). In this case, macroscopic and microscopic histochemical examinations showed actinomycete colonies consisting of yellow sulfur granules and basophilic filament aggregates. Fortu- 
nately, we were able to make a diagnosis based on a fineneedle aspiration biopsy; however, it can be very difficult to capture Actinomyces in small histological specimens $(18,19)$. As a result, many patients undergo hepatectomy due to the difficulty in detecting Actinomyces and distinguishing between Actinomyces abscesses and malignant tumors. Recently, new molecular genetic methods, such as polymerase chain reaction for 16s ribosomal RNA (rRNA) sequencing and fluorescence in situ hybridization, have been reported to be useful for obtaining a more rapid and accurate identification in reference or research laboratories (20). It has also been reported that these new methods have a higher rate of detection of bacteria than conventional cultivation methods in analyses of infectious pleurisy (21). We also analyzed the bacterial flora of the patient's pleural effusion obtained via the thoracic tube according to the clone library method using amplified fragments of the 16s rRNA gene; however, we were unable to detect any bacteria.

The recommended treatment for actinomycosis consists of intravenous penicillin for four weeks followed by oral penicillin for 12 months $(1,12,22)$. Prolonged treatment is also recommended due to the high rate of recurrence. We chose $\mathrm{ABPC} / \mathrm{SBT}$ at the beginning of treatment and later changed to EM based on our suspicion of an allergy to ABPC/SBT. Actinomyces are sensitive to a wide range of antimicrobial agents, including $\beta$-lactams, doxycycline, clindamycin, EM and clarithromycin, and the risk of developing resistance to these drugs is very low $(23,24)$. Therefore, EM is usually suitable for use in patients who are allergic to penicillin. However, the present patient's condition rapidly worsened after changing from ABPC/SBT to EM. Although the reason for this observation is unclear, the period of ABPC/SBT treatment and/or dose of EM may have been insufficient in this case.

Actinomyces grows more invasively than other bacteria and tends to spread locally across anatomic barriers by producing proteolytic enzymes $(10,25)$. However, it is uncommon for hepatic actinomycosis to infiltrate to neighboring organs. To our knowledge, there are only four case reports of direct invasion across the diaphragm $(24,26)$, two of which involved the pericardium, causing acute cardiac tamponade $(19,27)$. Moreover, another reported case involved a cutaneous fistula resulting from an actinomyces hepatic abscess (17). It is interesting that, in most cases, direct infiltration of hepatic actinomycosis to the neighboring organs occurred four weeks after treatment in spite of the use of correct antibiotics $(19,27)$. Therefore, physicians should carefully observe the patient at this period.

In conclusion, we herein reported a case of primary hepatic actinomycosis diagnosed on a needle aspiration biopsy. In this case, CEUS was very useful in evaluating the status of the disease. Although the hepatic abscesses initially rapidly improved following the administration of antibiotics, they later returned, involving both the right diaphragm and thorax. In cases of primary hepatic actinomycosis, the selection of antibiotics and period of treatment should be consid- ered carefully.

The authors state that they have no Conflict of Interest (COI).

\section{References}

1. Wong VK, Turmezei TD, Weston VC. Actinomycosis. BMJ 343 : d6099, 2011.

2. Putman HC Jr, Dockerty MB, Waugh JM. Abdominal actinomycosis; an analysis of 122 cases. Surgery 28: 781-800, 1950.

3. Russo TA. Agents of actinomycosis. In: Principles and Practice of Infectious Diseases. 7th ed. Mandell GL, Bennett JE, Dolin R, Eds. Elsevier Churchill Livingstone, London, 2010: 3209-3219.

4. Meade RH 3rd. Primary hepatic actinomycosis. Gastroenterology 78: $355-359,1980$.

5. Brown JR. Human actinomycosis. A study of 181 subjects. Hum Pathol 4: 319-330, 1973.

6. Sugano S, Matuda T, Suzuki T, et al. Hepatic actinomycosis: case report and review of the literature in Japan. J Gastroenterol 32: 672-676, 1997.

7. Sharma M, Briski LE, Khatib R. Hepatic actinomycosis: an overview of salient features and outcome of therapy. Scand J Infect Dis 34: 386-391, 2002.

8. Weese WC, Smith IM. A study of 57 cases of actinomycosis over a 36-year period. A diagnostic 'failure' with good prognosis after treatment. Arch Intern Med 135: 1562-1568, 1975.

9. Tambay R, Cote J, Bourgault AM, Villeneuve JP. An unusual case of hepatic abscess. Can J Gastroenterol 15: 615-617, 2001.

10. Yu CY, Chang WC, Gao HW, Chao TY, Huang GS, Hsieh CB. Metastatic hepatic actinomycosis. Am J Med 123: e9-e11, 2010.

11. Wayne MG, Narang R, Chauhdry A, Steele J. Hepatic actinomycosis mimicking an isolated tumor recurrence. World J Surg Oncol 9: 70, 2011.

12. Chen LW, Chang LC, Shie SS, Chien RN. Solitary actinomycotic abscesses of liver: report of two cases. Int J Clin Pract 60: 104107, 2006.

13. Liu CH, Yu CY, Catalano OA, Mueller PR. Radiological reasoning: imaging differentiation of a solitary hepatic mass. AJR Am J Roentgenol 6: S57-S61, 2008.

14. Wong JJ, Kinney TB, Miller FJ, Rivera-Sanfeliz G. Hepatic actinomycotic abscesses: diagnosis and management. AJR Am J Roentgenol 186: 174-176, 2006.

15. Catalano O, Sandomenico F, Raso MM, Siani A. Low mechanical index contrast-enhanced sonographic findings of pyogenic hepatic abscesses. AJR Am J Roentgenol 182: 447-450, 2004.

16. Kim TK, Jang HJ, Wilson SR. Benign liver masses: imaging with microbubble contrast agents. Ultrasound Q 22: 31-39, 2006.

17. Saad M, Moorman J. Images in clinical medicine. Actinomyces hepatic abscess with cutaneous fistula. N Engl J Med 353: e16, 2005.

18. Yamashita H, Fukuchi T, Ito D, et al. A case of hepatic actinomycosis diagnosed by thin needle aspiration biopsy successfully treated with antibiotics. Nihon Shokakibyo Gakkai Zasshi (Japanese Journal of Gastroenterology) 104: 698-702, 2007 (in Japanese, Abstract in English).

19. Sakaguchi Y, Isowa N, Nakazaki H, et al. Acute cardiac tamponade caused by the extension of multiple hepatic actinomycotic abscesses. Intern Med 51: 305-308, 2012.

20. Hansen JM, Fjeldsoe-Nielsen H, Sulim S, Kemp M, Christensen JJ. Actinomyces species: A danish survey on human infections and microbiological characteristics. Open Microbiol J 3: 113-120, 2009.

21. Kawanami T, Fukuda K, Yatera K, Kido M, Mukae H, Taniguchi $\mathrm{H}$. A higher significance of anaerobes: the clone library analysis of bacterial pleurisy. Chest 139: 600-608, 2011. 
22. Felekouras E, Menenakos C, Griniatsos J, et al. Liver resection in cases of isolated hepatic actinomycosis: case report and review of the literature. Scand J Infect Dis 36: 535-538, 2004.

23. Brook I. Actinomycosis: diagnosis and management. South Med J 101: 1019-1023, 2008.

24. Smith AJ, Hall V, Thakker B, Gemmell CG. Antimicrobial susceptibility testing of Actinomyces species with 12 antimicrobial agents. J Antimicrob Chemother 56: 407-409, 2005.

25. Kasano Y, Tanimura H, Yamaue H, Hayashido M, Umano Y. He- patic actinomycosis infiltrating the diaphragm and right lung. Am J Gastroenterol 91: 2418-2420, 1996.

26. Islam T, Athar MN, Athar MK, Usman MH, Misbah B. Hepatic actinomycosis with infiltration of the diaphragm and right lung: a case report. Can Respir J 12: 336-337, 2005.

27. Acevedo F, Baudrand R, Letelier LM, Gaete P. Actinomycosis: a great pretender. Case reports of unusual presentations and a review of the literature. Int J Infect Dis 12: 358-362, 2008.

(C) 2014 The Japanese Society of Internal Medicine http://www.naika.or.jp/imonline/index.html 